\title{
Assessment of the severity of obstructive sleep apnea syndrome through analysis of desaturations and apnea and hypopnea index: A review article
}

\author{
Amanda Bastos Lira ${ }^{1 *}$, Célio Fernando de Sousa Rodrigues ${ }^{2}$ and Fabiano Timbó Barbosa ${ }^{2}$ \\ ${ }^{1}$ Medical Sleep and ENT of Santa Casa Hospital in Maceió, Alagoas, Brazil \\ ${ }^{2}$ University of Alagoas, Brazil
}

\begin{abstract}
Purpose: The Obstructive Sleep Apnea Syndrome (OSAS) has been receiving great attention due to the systemic repercussions that can cause, especially to the cardiovascular system. Cycles of hypoxia caused by the obstruction of the upper airways during sleep, generate a change in the $\mathrm{O}_{2}$ oxidative chain increasing the production of free radicals, oxygen species highly reactive with other molecules. The severity of OSAS is assessed only by the quantification of these respiratory events (apnea and hypopnea index - AHI). In order to find new criteria for the definition of OSAS severity, a systematic review was made in the literature in search of other parameters for a better clinical evaluation of OSAS.
\end{abstract}

Methods: The literature search was conducted in 3 different databases (PubMed, LILACS and Scielo) using as descriptors the terms "obstructive sleep apnea", "apnea and hypopnea index" and "desaturations". We found 79 articles from which the titles and abstracts were read, but only 6 were included to read the texts and analyzes.

Results: The main pathophysiological consequences of OSAS occur due to insufficient supply of $\mathrm{O}_{2}$ caused by apnea and hypopnea. However, these events may have a short or long duration provided they have a minimum duration of 10 seconds. Events with prolonged duration and consequently with greater chances of deep desaturation may paradoxically lead to a decrease in AHI.

Conclusion: Quantification of desaturations as well as the depth level of $\mathrm{O}_{2}$ drops should be valued and may help to assess the real severity of OSAS in association with AHI.

To our knowledge, this is the first described case of spontaneous regression of squamous cell carcinoma of sinonasal origin.

\section{Introduction}

Among sleep disorders, the Obstructive Sleep Apnea Syndrome (OSAS) has been gaining prominence due to the systemic repercussions that it can cause, especially to the cardiovascular system, being an important cause of morbidity and mortality [1]. Cycles of hypoxia, caused by episodes of total obstruction (apnea) or partial obstruction (hypopnea) of the upper airways during sleep followed by reoxygenation, cause a change in the oxidative balance due to the insufficient supply of oxygen. This oxidative stress increases the number of free radicals, which are highly reactive species of oxygen molecules capable of reacting with other molecules, including proteins, altering their functions, being the main mechanism responsible for vascular endothelial injury and consequent cardiovascular complications [2].

All-night polysomnography is the necessary examination for the diagnosis and definition of OSAS severity, as it determines the rate of apnea and hypopnea (AHI), that is the number of these respiratory events per hour of sleep [3]. Thus, recurrence of respiratory events associated with frequent desaturations, and sleep surface and fragmentation are common findings in the polysomnography of patients with OSAS [4]. However, this is an examination of high cost and limited availability, mainly for the public health. Other alternatives have been proposed, especially as a screening for OSAS, such as pulse oximetry, since it is a low cost and easily performed technique, which is based mainly on periods of falls in $\mathrm{O}_{2}$ concentrations [3].
A task force was instituted by the American Academy of Sleep Medicine (AASM) in 1999 to establish the diagnostic criteria for OSAS, based on clinical and complementary aspects. Patients with OSAS should present diurnal or nocturnal symptoms, and at least 5 respiratory events (apnea/hypopnea) per hour of sleep ( $\mathrm{AHI} \geq 5 / \mathrm{h}$ ) demonstrated through polysomnography [5]. These criteria were revised and modified in 2005 , requiring the presence of $A+B+D$ or $C+D$ criteria (Table 1) [6]. The severity of OSAS is determined by three types of AHI ranges. Individuals with $\mathrm{AHI} \geq 5 / \mathrm{h}$ and $<15 / \mathrm{h}$ are considered mild. Those with $\mathrm{AHI} \geq 15$ and $\leq 30 / \mathrm{h}$ have moderate disease severity; and those with $\mathrm{AHI}>30 / \mathrm{h}$ are considered severe. Therefore, the severity of OSAS is evaluated only by AHI (Table 2) [5]. The American Academy of Sleep Medicine Scoring Manual recommended definition requires that changes in flow be associated with a $3 \%$ oxygen desaturation or a cortical arousal, but allows an alternative definition that requires association with a $4 \%$ oxygen desaturation without consideration of

Correspondence to: Amanda Bastos Lira, Medical Sleep and ENT of Santa Casa Hospital in Maceió, Alagoas, Brazil, Tel: +55(82)3327-2357/ +55(82)988881020; E-mail: ablira@hotmail.com

Key words: sleep apnea, apnea and hypopnea index, desaturations

Received: September 15, 2017; Accepted: October 26, 2017; Published: October 31, 2017 
Table 1. Diagnostic criteria for OSAS in adults according to the American Academy of Sleep Medicine, 2005. Source: The international classification of sleep disorders: Diagnostic and coding manual. Westchester American Academy of Sleep Medicine, 2005.

CRITERIA : $(\mathrm{A}+\mathrm{B}+\mathrm{D})$ or $(\mathrm{C}+\mathrm{D})$

A. At least one complaint:

- Unintended sleep episodes during wakefulness, excessive daytime sleepiness, unrepairable sleep, fatigue, or insomnia;

- Waking up with breathing pauses, gagging or choking;

- Companion report loud snoring and / or breathing pauses during sleep.

B. Polysomnography: $\geq 5$ respiratory events / hour of sleep (apnea, hypopnea, arousal related to respiratory effort). Evidence of respiratory effort throughout or part of each event.

C. Polysomnography: $\geq 15$ respiratory events / hour of sleep (apnea, hypopnea, arousal related to respiratory effort). Evidence of respiratory effort throughout or part of each event.

D. The disorder can not be better accounted for by other sleep disorders, medical or neurological diseases, use of medications, or use substances.

Table 2. Determination of OSAS severity by the quantification of AHI, according to the American Academy of Sleep Medicine, 1999. Source: Sleep-related breathing disorders in adults: recomendations for syndrome definition and measurement techniques in clinical research. Sleep: American Academy of Sleep Medicine, 1999.

\begin{tabular}{|c|c|}
\hline OSAS Severity & AHI \\
\hline MILD & $\geq 5$ and $<15 /$ hora \\
\hline MODERATE & $\geq 15$ and $\leq 30 /$ hora \\
\hline SEVERE & $>30 /$ hora \\
\hline
\end{tabular}

cortical arousals. Depending on which definition is used, the AHI may be considerably different in a given individual [7].

Considering that in addition to lowering airflow, it is also an absolute criterion for defining any respiratory event the duration of airflow, which should be at least 10 seconds (Table 3) [8]. Thus, There are short and long respiratory events. Longer-lasting events are more likely to result in more significant declines in $\mathrm{O}_{2}$ concentrations and consequent more significant desaturations of oxyhemoglobin, resulting in more deleterious effects on health. So, it is possible that individuals with low AHI, but with long-term respiratory events and, therefore, with more severe oxyhemoglobin desaturations, may suffer more hemodynamic damage than individuals with AHI, but with short respiratory events and milder desaturations of oxyhemoglobin. Kulkas et al. [9] researched new parameters to evaluate the OSAS severity, relating duration and morphology of obstructive events during sleep. They found that subjects with similar AHI had different degrees of upper airway obstruction with different levels of oxyhemoglobin desaturation. Most intriguingly, short respiratory events could raise AHI, but they did not necessarily lead to significant falls in $\mathrm{O}_{2}$ levels. Thus, when using only AHI as a criterion of OSAS severity, one may be underestimating cases with greater severity, but with low AHI, or otherwise, overestimating less severe cases, but with high AHI.

In order to find new criteria for the definition of OSAS severity that support AHI, and therefore together, can more accurately determine the true severity of the disease, a systematic review was performed in the literature in search of other parameters that could contribute to a better clinical evaluation of OSAS.

\section{Methods}

\section{Extraction of data}

Data search in the literature was performed by 3 researchers independently and in 3 different databases (PubMed, LILACS and Scielo), since january to october 2017. The terms "obstructive sleep apnea "," apnea and hypopnea index "and" desaturations "were used as descriptors, according to the following search strategies: (" Obstructive sleep apnea "or" sleep apnea, obstructive “ "All Fields] AND” apnea "[All Fields] AND" obstructive "[All Fields]) OR" obstructive sleep apnea"] AND "apnea” [All Fields])) AND ("apnoea" [All Fields] OR "apnea" [MeSH Terms] OR "apnea" [All Fields] AND "topic" [All Fields] OR "abstracting" [All Fields] AND "indexing" [All Fields] AND "topic" Fields]) AND desaturations [All Fields]. In this way, 79 articles were found from which the titles and abstracts were read. Those who were considered of interest to the research were selected to read the texts in full.

\section{Eligible studies}

The articles that were selected for this research should follow the following inclusion criteria: (1) prospective or retrospective studies in adult humans diagnosed with OSAS; (2) analyzes of desaturations in the evaluation of OSAS beyond AHI. Unpublished studies or those that did not meet the above criteria were excluded. Thus, 79 articles were found, but only 10 were included for complete reading of the texts. Each researcher independently carried out the readings of the 10 articles in their entirety, of which 6 were unanimously considered among the researchers of interest to the research and therefore, under which the analyzes should be made [3,9-14]. The main results of each article were extracted and these were compared by the authors, with agreement among them. However, it was not possible to carry out statistical analysis due to the heterogeneity of the variables.

\section{Results and discussion}

The treatment of OSAS depends fundamentally on the severity of the disease. All efforts should be made to try to minimize the harmful effects of the disease primarily related to the cardiovascular system. $\mathrm{O}_{2}$ deficiency caused by obstruction of the upper airways during sleep generates intermittent periods of hypoxia that alter oxidative metabolism. This oxidative stress produces an imbalance between pro-oxidant and anti-oxidant factors, increasing the reactive $\mathrm{O}_{2}$ species which have one or more unpaired electrons in their outer orbit, and because of that they are chemically reactive. These free radicals are products normally obtained from $\mathrm{O}_{2}$ metabolism during the cellular respiration process, through enzymatic and non-enzymatic antioxidant systems act to eliminate their excess [10]. However, there is an imbalance of this antioxidant capacity in OSAS, generating a greater quantity of these reactive species of the $\mathrm{O}_{2}$ that are able to interact with other molecules and to change their functions. In addition, they may

Table 3. Criteria for identifying apneas and hypopneas according to the American Academy of Sleep Medicine, 2007. Source: Iber C, Ancoli-Israel S, Chesson JRA et al (2007). The AASM manual for the scoring of sleep and associated events: rules, terminology and technical specifications. Westchester: IL: American Academy of Sleep Medicine, $1^{\text {st }}$ Ed.

\begin{tabular}{|l|l|l|}
\hline Respiratory Events & Definition & Duration \\
\hline APNEA & $\begin{array}{l}\text { Airflow excursion drop by } \\
\text { temperature signal } \geq 90 \% .\end{array}$ & $\geq 10$ seconds \\
\hline & $\begin{array}{l}\text { Reduction of airflow } \\
\text { excursion by nasal pressure } \\
\text { signal } \geq 30 \% \text { of baseline } \\
\text { associated with fall } \geq \\
4 \% \text { of oxygen saturation } \\
\text { (recommended criteria }-\end{array}$ & \\
rule A); \\
HYPOPNEA & $\begin{array}{l}\text { OR } \\
\text { Fall } \geq 50 \% \text { of the nasal } \\
\text { pressure signal associated } \\
\text { with fall } \geq 3 \% \text { of oxygen } \\
\text { saturation or an awakening } \\
\text { (alternative criterion - rule }\end{array}$ & \\
B). \\
\hline
\end{tabular}


also activate proinflammatory factors with cytokine production, such as interleukins 6 and 8 and tumor necrosis factor, which are involved in the genesis of systemic arterial hypertension and atherosclerosis in OSAS [2].

Thus, the main pathophysiological consequences of OSAS occur due to the insufficient supply of $\mathrm{O}_{2}$. Quantification of desaturations as well as the depth level of $\mathrm{O}_{2}$ drops should be valued and may help to assess the real severity of OSAS in association with AHI such as George et al. [11]. In that study, the authors used a computerized algorithm to detect falls in $\mathrm{O}_{2}$ saturation greater than $3 \%$, evaluating the saturation in two moments: at the end of inspiration and at the end of expiration. The program scanned through time series between peaks and depressions of $\mathrm{O}_{2}$ saturation. A file with all events was created and correlated to the stages of non-REM and REM sleep, indirectly determining an AHI. Each patient underwent a conventional polysomnography to determine the traditional AHI through the visual reading of the technician to mark the respiratory events, and then they were also submitted to analysis through the computerized algorithm proposed for the determination of indirect AHI based on desaturations. There was a strong correlation between the 2 methods, suggesting that AHI can be easily estimated by the number of desaturations, as well as being a more easily analyzed method and not subject to human error (Table 4).

Gyulay et al. [12] made a comparison between polysomnography and home oximetry for the diagnosis of OSAS. Analyzes of pulse oximetry-based desaturations were highly specific for the disease, when desaturations were greater than $4 \%$ and generated an index of desaturations $\geq 15 / \mathrm{h}$ as they were equated to an AHI $\geq 15 / \mathrm{h}$. The authors concluded that home oximetry is applicable as a screening for OSAS (Table 4).

Guilleminault et al. [13] evaluated lean OSAS patients through baseline polysomnography, analyzing all respiratory events according to the criteria established by the AASM 1999 (rule C), and by the variation of the criteria revalidated by the AASM in 2007, in which there are 2 options for defining hypopnea (rules A and B), according to the level of $\mathrm{O}_{2}$ desaturation or the presence of cortical arousal. According to rule A, hypopnea is considered when there is an airflow drop of at least $30 \%$, but only if it is associated with a desaturation $\geq 4 \%$. Rule $\mathrm{B}$, however, considers hypopnea when there is at least $50 \%$ drop in airflow accompanied by desaturation of $3 \%$ or an awakening. As can be observed, according to rule $\mathrm{B}$ there may or may not be $\mathrm{O}_{2}$ desaturation. Rule C of 1999 used only 1 criterion for hypopnea, and may or may not be associated with desaturations (drop $\geq 50 \%$ of airflow associated with desaturations of $\geq 3 \%$ or wakefulness). All 35 patients in the study had an $\mathrm{AHI}<15 / \mathrm{h}$ according to rule A. Rule B resulted in 11 subjects with AHI below 15 / hour, while by rule C no patient had AHI below 15/h.

Table 4. Human studies on OSAS assessment criteria beyond AHI.

\begin{tabular}{|c|c|}
\hline $\begin{array}{l}\text { Researches about evaluation of OSAS } \\
\text { beyond AHI }\end{array}$ & Main findings refer to desaturations \\
\hline George CF, et al. [11] & Estimation of AHI through desaturations \\
\hline Gyulay S, et al. [12] & Estimation of AHI through desaturations \\
\hline Guilleminault C, et al. [13] & $\begin{array}{l}\text { Variation of AHI according to the rule used } \\
\text { for marking hypopneas }\end{array}$ \\
\hline Kulkas A, et al. [14] & $\begin{array}{l}\text { Assessment of the severity and duration } \\
\text { of desaturations as parameters of OSAS } \\
\text { severity }\end{array}$ \\
\hline Schlotthauer G, et al. [3] & Estimation of AHI through desaturations \\
\hline Kulkas A, et al. [14] & $\begin{array}{l}\text { Desaturation events differs between } \\
\text { hypopnea and obstructive apnea events }\end{array}$ \\
\hline
\end{tabular}

Source: the authors
These results are intriguing because they show that depending on the rule used to label hypopneas, the value of AHI may be underestimated or overestimated. The authors concluded that desaturations are clearly important especially for the recognition of OSAS (Table 4).

Another very interesting research was carried out in 2013 by Kulkas et al. [9]. The authors highlighted the analysis of desaturations correlating them with the severity of OSAS through the determination of AHI. 160 patients with suspected OSAS were evaluated for the number, duration and morphology of respiratory events and desaturations, and not only by counting these events per hour of sleep as determined by AHI. They found that the prolonged duration of apnea and hypopnoea may paradoxically lead to a fall in AHI, although this has more serious health consequences than shorter events. The variety aspect of the duration of these events is not included in the traditional AHI. Another aspect to be considered is the morphology of $\mathrm{O}_{2}$ desaturation events that is also not considered in AHI. Deep and long respiratory events of $\mathrm{O}_{2}$ desaturation presumably contribute more to pathophysiological stress than mild and short events. In addition to AHI, the authors used 4 new evaluation parameters because of the suspicion of differences in severity of events in patients with similar AHI: severity of obstruction and desaturation, duration of obstruction and desaturation. There was a moderate correlation between $\mathrm{AHI}$ and the new severity parameters $(\mathrm{r} 2=0.581-0.689, \mathrm{p}<0.001)$. Since patients with similar AHI showed significant differences in values of obstruction severity parameters, this could provide different diagnoses of severity for OSAS patients. Thus, the severity of OSAS could be better estimated based on these new criteria (Table 4).

The nocturnal oximetry analyzes have been used as screening for potential diagnosis of OSAS, but the interpretation of the signal is highly dependent on the technician. Some studies have shown that when appropriate algorithms are used, nocturnal oximetry can be a really sensitive and specific method for OSAS. This was demonstrated by Schlotthauer et al. [3], in which an algorithm based on the empirical decomposition method for the detection of desaturations associated with OSAS was demonstrated, using signals obtained by pulse oximetry, in order to estimate the AHI as a mode similar to AHI obtained through polysomnography, but using only information on oxygen desaturations measured by oximetry. Only $\mathrm{O}_{2}$ falls of at least $3 \%$ and lasting for at least 10 seconds were considered equivalent to the occurrence of some respiratory events. The authors concluded that the algorithm used based on desaturations may contribute to the estimation of AHI (Table 4).

A recent study published by Kulkas et al. [14], in which the duration and depth of desaturations were evaluated in the respiratory events (apnea and hypopnea) of 395 patients undergoing an allnight polysomnography, which were divided into groups according to duration of desaturation events. For marking of hypopneas, the authors considered desaturations from $3 \%$. There was a statistically significant correlation between the time and depth of desaturations and the duration of apnea and hypopnea $(\mathrm{p}<0.001)$, and also observed that the events of desaturations caused by apneas were more significant than desaturations after hypopnea $(\mathrm{p} \leq 0.004)$. The authors concluded that the longer respiratory events generally induce more severe desaturations and therefore, more detailed analyzes of the severity of desaturations and duration of respiratory events should be performed for an adequate estimate of OSAS severity (Table 4).

The above studies point to the importance of desaturations as an attempt to diagnose OSAS, and only the studies by Kulkas et al. $[9,14]$ make some relation between the severity of OSAS and the number 
and degree of desaturation depth. There is evidence that the worst consequences of OSAS have a strong relationship with low levels of $\mathrm{O}_{2}$ concentration. However, more studies are needed to prove the correlation between falls in $\mathrm{O}_{2}$ concentrations and the severity of the disease.

\section{Conclusion}

The quantification of respiratory events per hour of sleep remains the only criterion recommended for the determination of OSAS severity, and although polysomnography provides information on the number and depth of desaturations, it is not yet a criterion used to determine the severity of the disease. There are some published studies that establish a relationship between desaturations and AHI, but we did not find studies that correlated AHI, desaturations and OSAS severity. Thus, it is necessary to establish new criteria that together with the AHI determine the real severity of OSAS.

\section{Acknowledgments}

The authors would like to thank the University of Alagoas/Brazil.

\section{References}

1. de Sousa Rodrigues CF, Lira AB (2015) Correlation between the severity of apnea and hypopnea sleep, hypertension and serum lipid and glycemic: a case control study. Eur Arch Otorhinolaryngol 272: 1509-1515. [Crossref]

2. Lira AB, Sousa-Rodrigues (2016) Evaluation of oxidative stress markers in obstructive sleep apnea syndrome and additional antioxidante therapy: a review article. Sleep Breath 20: 1155-1160. [Crossref]

3. Schlotthauer G, Di Persia LE, Larrateguy LD, Milone DH2 (2014) Screening of obstructive sleep apnea with empirical mode decomposition of pulse oximetry. Med Eng Phys 36: 1074-1080. [Crossref]
4. Yaganoglu M, Kayabekir M, Köse C (2017) SNORAP: A Device for the Correction of Impaired Sleep Health by Using Tactile Stimulation for Individuals with Mild and Moderate Sleep Disordered Breathing. Sensors. [Crossref]

5. American Academy Sleep Medicine Task Force (1999) Sleep-related breathing disorders in adults: recomendations for syndrome definition and measurement techniques in clinical research. Sleep 22 : 667-689. [Crossref]

6. American Academy Sleep Medicine (2005) The international classification of sleep disorders: diagnostic and coding manual. $\left(2^{\text {nd }}\right.$ edn) Westchester.

7. Kapur VK, Auckley DH, Chowdhuri S, Kuhlmann DC, Mehra R, et al. (2017). Clinical Practice Guideline for Diagnostic Testing for Adult Obstructive Sleep Apnea: An American Academy of Sleep Medicine Clinical Practice Guideline. J Clin Sleep Med 13: 479-504. [Crossref]

8. Iber C, Ancoli-Israel S, Chesson JRA (2007) The AASM manual for the scoring of sleep and associated events: rules, terminology and technical specifications. ( $1^{\text {st }}$ edn $)$ Westchester, IL: American Academy of Sleep Medicine.

9. Kulkas A, Tiihonen P, Julkunen P, Mervaala E, Töyräs J (2013) Novel parameters indicate significant diferences in severity of obstructive sleep apnea with patient having similar apnea-hypopnea index. Med Biol Eng Comput 51: 697-708. [Crossref]

10. Lavie L (2003) Obstructive sleep apnoea syndrome--an oxidative stress disorder. Sleep Med Rev 7: 35-51. [Crossref]

11. George CF, Millar TW, Kryger MH (1988) Identification and quantification of apneas by computer-based analysis of oxygen saturation. Am Rev Respir Dis 137: 1238-1240. [Crossref]

12. Gyulay S, Olson LG, Hensley MJ, King MT, Allen KM, et al. (1993) A comparison of clinical assessment and home oximetry in the diagnosis of obstructive sleep apnea. $\mathrm{Am}$ Rev Respir Dis 147: 50-53. [Crossref]

13. Guilleminault C, Hagen CC, Huynh NT (2009) Comparison of hypopnea definitions in lean patients with know obstructive sleep apnea hypopnea syndrome (OSAHS). Sleep Breath 13: 341-347. [Crossref]

14. Kulkas A, Duce B, Leppänen T, Hukins C, Töyräs J (2017) Severity of desaturation events differs between hypopnea and obstructive apnea events and is modulated by their duration in obstructive sleep apnea. Sleep Breath [Crossref]

Copyright: (C2017 Lira AB. This is an open-access article distributed under the terms of the Creative Commons Attribution License, which permits unrestricted use, distribution, and reproduction in any medium, provided the original author and source are credited. 